\title{
MOLAR DECÍDUO INFRAOCLUIDO: RELATO DE UM CASO RESTAURADO COM CIMENTO IONÔMERO DE VIDRO ENCAPSULADO, UM ANO DE ACOMPANHAMENTO
}

\author{
Ana Paula DORNELLAS ${ }^{1 *}$, Karla Dayanna Tavares CAVALCANTE ${ }^{1}$, Tamara Kerber TEDESCO ${ }^{1,2}$, \\ Isabela FLORIANO ${ }^{1,3}$ \& José Carlos IMPARATO ${ }^{1,4}$
}

\author{
1 Faculdade São Leopoldo Mandic. Campinas, São Paulo, Brasil. \\ 2 Universidade Ibirapuera. São Paulo, Brasil. \\ 3 Centro Universitário UNINOVAFAPI. Teresina, Piauí, Brasil. \\ 4 Universidade de São Paulo. São Paulo, Brasil. \\ *Autor para correspondência: anapauladornellas@hotmail.com
}

DOI: http://dx.doi.org/10.18571/acbm.179

\section{RESUMO}

A infraoclusão de dentes decíduos pode ocorrer devido a fusão anatômica do cemento dentário com o osso alveolar, situação que denominamos como anquilose dentária. Esse evento, comum em molares decíduos, pode causar prejuízo no desenvolvimento da oclusão. O objetivo desse trabalho foi relatar a reabilitação de um molar decíduo infra-ocluído utilizando cimento de ionômero de vidro modificado por resina encapsulado (Riva Ligh Cure, SDI, Brasil) e seu acompanhamento durante doze meses. Paciente de 9 anos, do sexo feminino, compareceu ao consultório acompanhado de sua mãe reclamando de um "buraco no dente". Após diagnostico de anquilose, foi realizada a restauração no elemento dentário em sessão única e realizado acompanhamento clínico e radiográfico durante doze meses. Ao final desse período observamos que o dente tratado permaneceu restaurado o que proporcionou a esfoliação das raízes do dente decíduo e consequentemente a erupção do dente permanente. Diante disso, concluímos que é possível tratar molares decíduos em infraoclusão com cimento de ionômero de vidro modificado por resina, no intuito de facilitar a realização do procedimento em sessão única e diminuir os riscos associados a um dente anquilosado não tratado precocemente.

Palavras Chave: Dentes decíduos; Odontopediatria; Anquilose dentária; Cimento de ionômero de vidro.

\section{ABSTRACT}

The infraocclusion of deciduous teeth may occur due to the anatomical fusion of the dental cementum with the alveolar bone, a situation we call dental ankylosis. This event, common in deciduous molars, may cause impairment in occlusion development. The objective of this work was to report the rehabilitation of an infra-occluded deciduous molar using encapsulated resinmodified glass ionomer cement (Riva Ligh Cure, SDI, Brazil) and its follow-up for twelve months. A 9-year-old female patient attended the office accompanied by her mother complaining about a "hole in the tooth". After diagnosis of ankylosis, restoration was performed on the dental element in a single session and clinical and radiographic follow-up was performed for twelve months. At the end of this period we observed that the treated tooth remained restored which provided the exfoliation of the roots of the deciduous tooth and consequently the eruption of the permanent tooth. Therefore, we conclude that it is possible to treat deciduous molar teeth in infraocclusion with resin-modified glass ionomer cement, in order to facilitate the single-session procedure and to reduce the risks associated with an early, untreated ankylosed tooth.

Keywords: Primary Dentition; Pediatric Dentistry; Dental ankylosis; Glass ionomer cement. 


\section{Biomedica Brasiliensia}

\section{Introdução}

A anquilose dentária resulta da fusão entre cemento e o osso alveolar (PONDURI, BIRNIE \& SANDRY, 2014). Mais prevalente em molares decíduos, ela pode resultar em má oclusão e até mesmo na retenção prolongada do dente decíduo e, por conta disso, impactar de maneira negativa a qualidade de vida do paciente (MISHRA et al 2010). Quando identificado precocemente, o cirurgião-dentista têm a oportunidade de minimizar a extensão dos problemas bucais futuros derivados de molares decíduos em infraoclusão (ALVES et al, 2011).

Distúrbios oclusais causados por infraoclusão podem ser prevenidos por meio de aumento de coroa e contatos interproximais com materiais adesivos (ARHAKIS \& BOUTIOU, 2016). Diversos estudos ressaltam a importância do restabelecimento oclusal nos casos de anquilose leve com técnicas restauradoras indiretas (CAVANAUGH, 1994 e SABATINI 2010) e/ou restaurações com resina composta (MOURA et al 2015).

Para o melhor de nosso conhecimento, não há estudos que tenham relatado a reabilitação de dentes anquilosados com uso de cimento de ionômero de vidro, mesmo sendo considerado o material de escolha para uma variedade de procedimentos clínicos. O cimento de ionômero de vidro modificado por resina, em especial, tem se destacado por apresentar adesão aos tecidos mineralizados, biocompatibilidade, possuir coeficiente de expansão térmica semelhante ao dente, liberação de flúor e maior resistência frente aos esforços mastigatórios (SILVA et al, 2011; BERG, 2002).

Diante disso, o objetivo desse estudo é descrever o tratamento adotado para um primeiro molar decíduo infraocluído com restauração de cimento de ionômero de vidro fotopolimerizável encapsulado. Além disso, o relato demonstra a utilização de uma técnica alternativa com a utilização de uma banda ortodôntica no intuito de facilitar os procedimentos de restauração direta e minimizar os procedimentos de acabamento envolvidos na colocação de grandes restaurações.

\section{Relato de caso}

Para esse relato de caso, seguiremos as diretrizes CARE (2013) de verificação de informações a serem consideradas durante a redação de um relato de caso.

\subsection{Informações do paciente}

Uma criança de nove anos de idade, sexo feminino, caucasiana, compareceu ao consultório odontopediátrico acompanhada de sua mãe. Queixou-se do "buraco" presente entre os dentes de baixo, que aparecia ao sorrir. Sua história médica não apresentou nenhum tipo de alteração sistêmica que tenha relevância para esse relato.

\subsection{Achados clínicos e radiográficos}

Ao exame clínico, a paciente apresentou dentição mista, ausência de lesões de cárie, molares permanentes em relação de classe 1 de Angle, mordida cruzada unilateral direita entre canino decíduo superior direito (53) e canino decíduo inferior direito (83) e primeiro molar decíduo direito (84) em infraoclusão e sem mobilidade (Figura 1A)

O exame radiográfico periapical apontou ausência do ligamento periodontal e fusão entre o cemento e osso alveolar no elemento 84 , além de posição da superfície oclusal próxima ao ponto de contato com os dentes adjacentes (Figura 1B). 

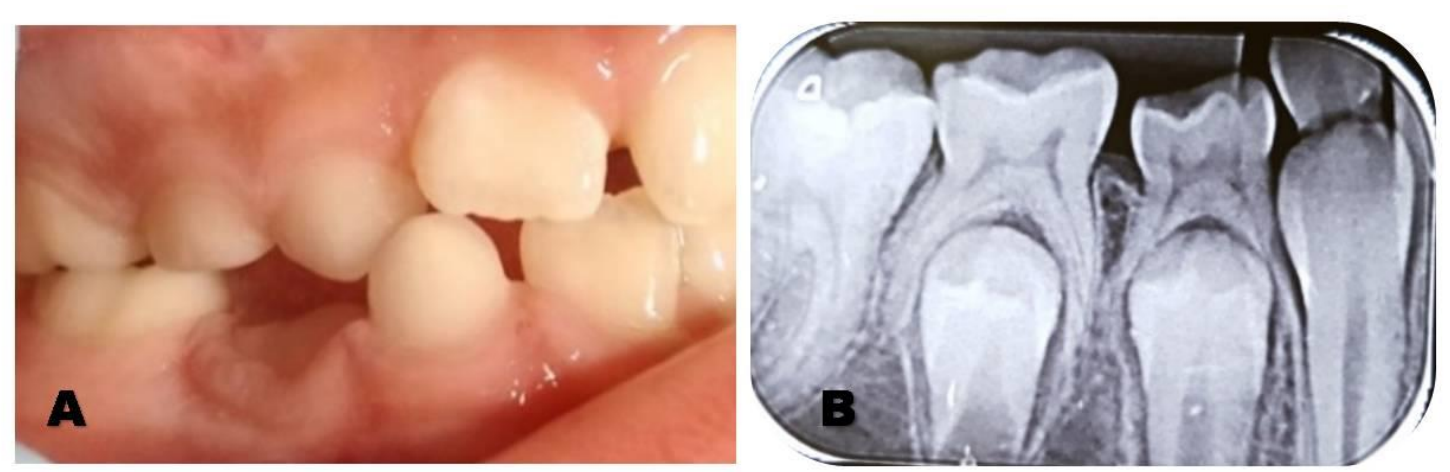

Figura 1: (A) Fotografia inicial, mostrando o elemento 84 em infraoclusão e caninos com mordida cruzada. (B) Radiografia periapical inicial, com o dente 84 apresentando ausência de ligamento periodontal, fusão entre o cemento e osso alveolar e posição da superfície oclusal próxima ao ponto de contato com os dentes adjacentes.

Considerando os aspectos clínicos e radiográficos chegou-se ao diagnóstico de anquilose dentária grau moderado, de acordo com o índice de anquilose de Brearley \& McKibben (1973).

\subsection{Intervenção terapêutica}

Após profilaxia dentária profissional, selecionou-se e cimentou-se banda ortodôntica no dente 84 com cimento de ionômero de vidro encapsulado fotopolimerizável (Riva Ligh Cure, SDI, Brasil) (Figura 2A). Em seguida, removeu-se a banda e procedeu-se ao acabamento e polimento da restauração (figura $2 \mathrm{~B}$ ).
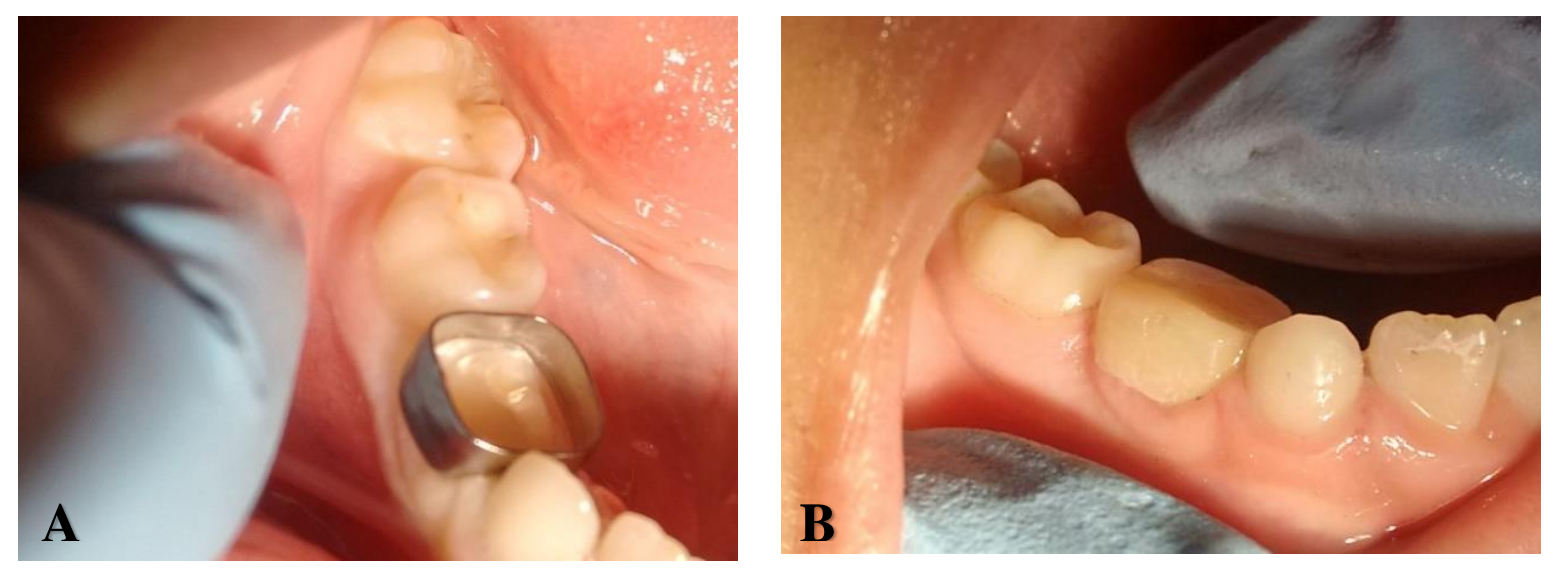

Figura 2: (A) Banda ortodôntica posicionada no dente anquilosado. (B) Aspecto final após a remoção da banda.

\subsection{Seguimento clínico e resultados}

Seis meses depois foi realizado novo exame clínico e radiográfico. Clinicamente, a restauração não apresentou falhas ou desgastes. Radiograficamente, observou-se o dente 84 em processo de rizólise (Figura 3A). Com um ano do procedimento, o dente 84 demonstrou restauração em bom estado e mobilidade dentária (Figura 3B). 

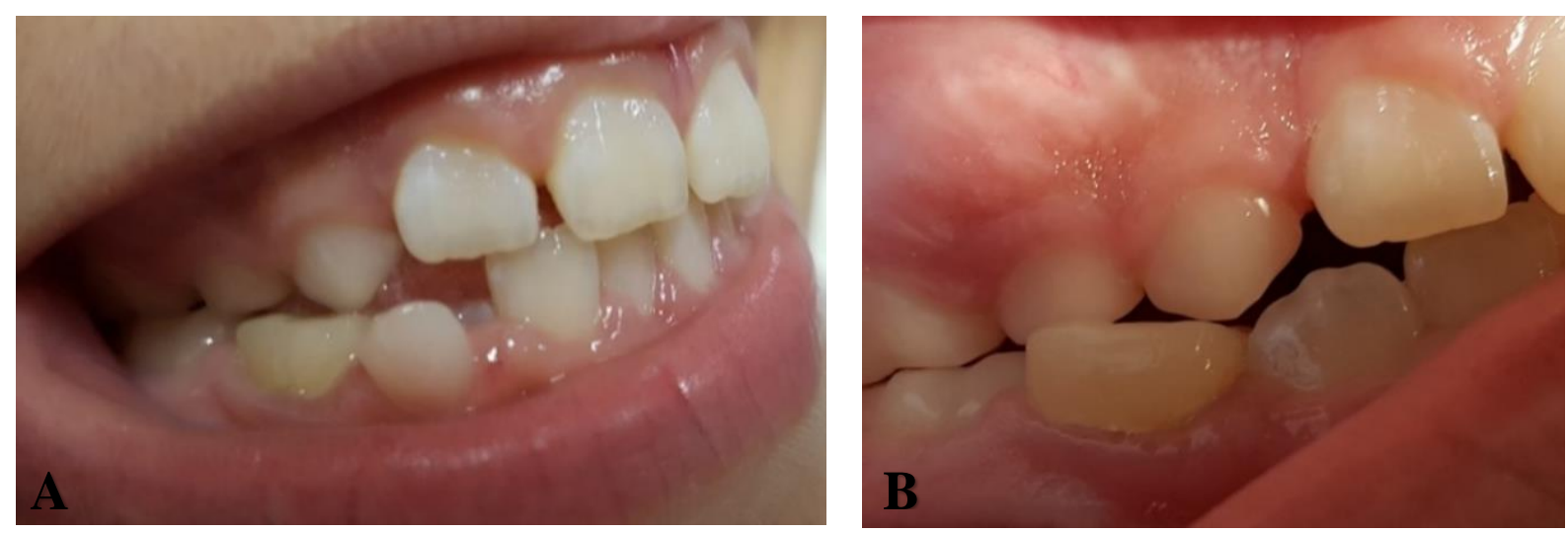

Figura 3: Aspecto clínico da restauração (A) após 06 meses, (B) após 12 meses. Observa-se restauração íntegra.

O exame radiográfico periapical mostrou reabsorção das raízes e a radiografia panorâmica apontou o primeiro pré-molar inferior direito (44) em estágio 8 de Nolla (Figura 4). A exodontia do elemento 84 foi realizada.
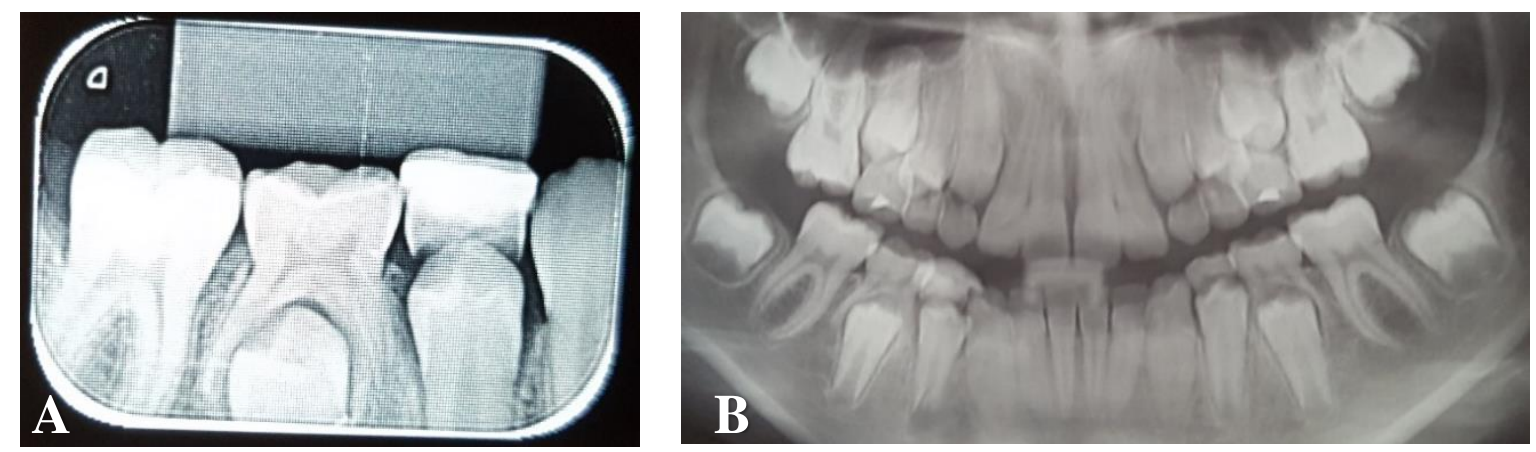

Figura 4: (A) Radiografia periapical, demonstra a reabsorção das raízes do elemento 84 (B) Radiografia panorâmica, observa-se o elemento 44 em estágio 8 de Nolla.

\section{Discussão}

Baseados em estudo de Lavanaugh e Croll (1994) nos propomos a investigar se seria possível a reabilitação em uma única sessão de um dente decíduo anquilosado, utilizando cimento de ionômero de vidro modificado por resina encapsulado e uma banda ortodôntica. O principal achado após doze meses de acompanhamento foi a boa adesão e resistência do material frente aos esforços mastigatórios e consequentemente a rizólise do dente decíduo, proporcionando a erupção do elemento dentário permanente sem necessidade de remoção cirúrgica do elemento decíduo.

No caso clínico apresentado, a paciente tinha 9 anos de idade e queixava-se de um "buraco" entre os dentes que aparecia ao sorrir. A conduta clínica foi realizada levando em consideração a severidade da infraoclusão, idade da criança e a distância entre o município da família ao consultório odontológico. Lourenço Neto et al. (2011), relataram bons resultados para a reabilitação de molares decíduos infraocluídos com restauração indireta com resina composta como melhor adaptação marginal frente às restaurações diretas e menor chance de infiltrações. Entretanto, para a realização desta técnica a peça foi confeccionada a partir do modelo de gesso do paciente, o que demandaria mais sessões e deslocamentos para a mãe e a paciente do caso clínico descrito. 
Encontramos na literatura outros tipos de tratamentos, desde os mais conservadores, como o acompanhamento clínico e radiográfico sem intervenção até a exodontia do elemento dentário e o uso de mantenedores de espaço (Pitoni et al., 2006). A técnica aqui descrita mostrou-se eficaz e é de fácil realização pelo cirurgião-dentista, dispensando materiais complexos. Com isso, preservamos os espaços do arco dentário e a oclusão normal do paciente até o dente sucessor permanente irromper. No entanto, não se sabe se isto teria acontecido caso o tratamento de restabelecimento oclusal não houvesse sido realizado.

Especulamos que a utilização do cimento ionômeros de vidro modificado por resina encapsulado tenha sido uma alternativa eficaz para a restauração do dente decíduo anquilosado. Estudo clínico randomizado de boca dividida realizado por Freitas et al. (2018) comparou ionômeros encapsulados e ionômeros manipulados manualmente e os cimentos encapsulados apresentaram uma taxa de falha de $24 \%$ comparado ao manipulado de $42 \%$. O estudo de Molina et al. (2013), corrobora com esse resultado e indica que o ionômero de vidro encapsulado também pode contribuir para um aumento nas taxas de sobrevida das restaurações de ionômero de vidro para Tratamento Restaurador Atraumático em dentes decíduos e permanentes.

Acreditamos, portanto, que apesar do aumento da aplicabilidade clínica e melhora das propriedades dos cimentos de ionômero de vidro (Silva et al 2011), neste estudo atingimos um resultado satisfatório utilizando esse material devido a presença do sucessor permanente, ou seja, a permanência do material seria por um período de tempo curto. Devido a essa característica, sugerimos novos estudos com tempo de acompanhamento maior em dentes anquilosados decíduos restaurados com cimento de ionômero de vidro modificado por resina.

\section{Conclusão}

Concluímos que a utilização de banda ortodôntica e cimento de ionômero de vidro fotopolimerizável encapsulado foi uma boa opção para a reabilitação de dentes decíduos com anquilose. Além disso, permitiu a rizólise do dente decíduo e posterior extração.

\section{Referências}

ALVES M.S.C., LEITE T.H.M., VIEIRA D.R.P., CRUZ M.C.F.N., ALVES C.M.C. Diagnosis and treatment of severe dentoalveolar ankylosis in primary molars: case report. Rev Odontol UNESP. Vol .40(3), p. 154-159, 2011.

ARHAKIS A. \& BOUTIOU E. Etiology, Diagnosis, Consequences and Treatment of Infraoccluded Primary Molars. Open Dent J. Vol. 10, p. 714-719, 2016.

BERG JH. Glass ionomer cements. Pediatr Dent Vol. 24, p. 430-438, 2002.

BREARLEY LJ, MCKIBBEN DH JR. Ankylosis of primary molar teeth. I. Prevalence and characteristics. ASDC J Dent Child. Vol. 40, p. 54-63, 1973.

CAVANAUGH, RR.; CROLL, TP. Resin-bonded ceramic onlays for retained primary molars with infraocclusion. Quintessence International. Vol. 25(7), p. 459-463, 1994.

FREITAS MCCA, FAGUNDES TC, MODENA KCS, CARDIA GS, NAVARRO MFL.Randomized clinical trial of encapsulated and hand-mixed glass-ionomer ART restorations: one-year follow-up. J Appl Oral Sci .Vol. 26, 2018. 
LOURENÇO NETO N, GURGEL CV, KOBAYASHI TY, BIJELLA MFB, OLIVEIRA TM, MACHADO MAAM, RIOS D. Uso de Restaurações Indiretas na Correção da Infra-Oclusão: Relato de Caso. Rev Odontol Bras Central. Vol. 20, 2011.

MISHRA SK, JINDAL MK, SINGH RP, STARK TR, HASHMI GS. Submerged and Impacted Primary Molars. International Journal of Clinical Pediatric Dentistry. Vol. 3, p. 211-213, 2010.

MOLINA GF, CABRAL RJ, MAZZOLA I, BRAIN LASCANO L, FRENCKEN JE. Mechanical performance of encapsulated restorative glass-ionomer cements for use with Atraumatic Restorative Treatment (ART). J Appl Oral Sci. Vol. 21(3), p. 243-9, 2013.

MOURA MS, PONTES AS, BRITO MHSF, MOURA LD, LIMA MDM, SIMPLICIO AHM. Restorative Management of Severely Ankylosed Primary Molars . Journal of Dentistry for Children. Vol. 82 (1), 2015.

PITONI CM, CALDO-TEIXEIRA AS, ALMEIDA ICS, VIEIRA RS. Restaurações Adesivas Indiretas Opção Clínica para Molares Decíduos em Infra-Oclusão. R. Fac. Odontol. Porto Alegre, Porto Alegre, v. 47, p. 39-42, 2006.

PONDURI S, BIRNIE DJ, SANDY JR. Infraocclusion of secondary deciduous molars--an unusual outcome. J Orthod. Vol. 36(3), p. 186-9, 2009.

RILEY ET AL. Care guidelines for cases report : explanation and elaboration document. Journal of Clinical Epidemiology, 2017.

SABATINI C.; An Alternative Approach to the Transitional Rehabilitation of Infra-Occluded Primary Second Molars. journal compilation. Vol. 22(6), 2010.

SILVA FWGP.; Queiroz AM.; Freitas AC, Assed S. Utilização do ionômero de vidro em odontopediatria. Odontol. Clín.-Cient., Recife, Vol. 10(1), p.13 - 17, 2011. 